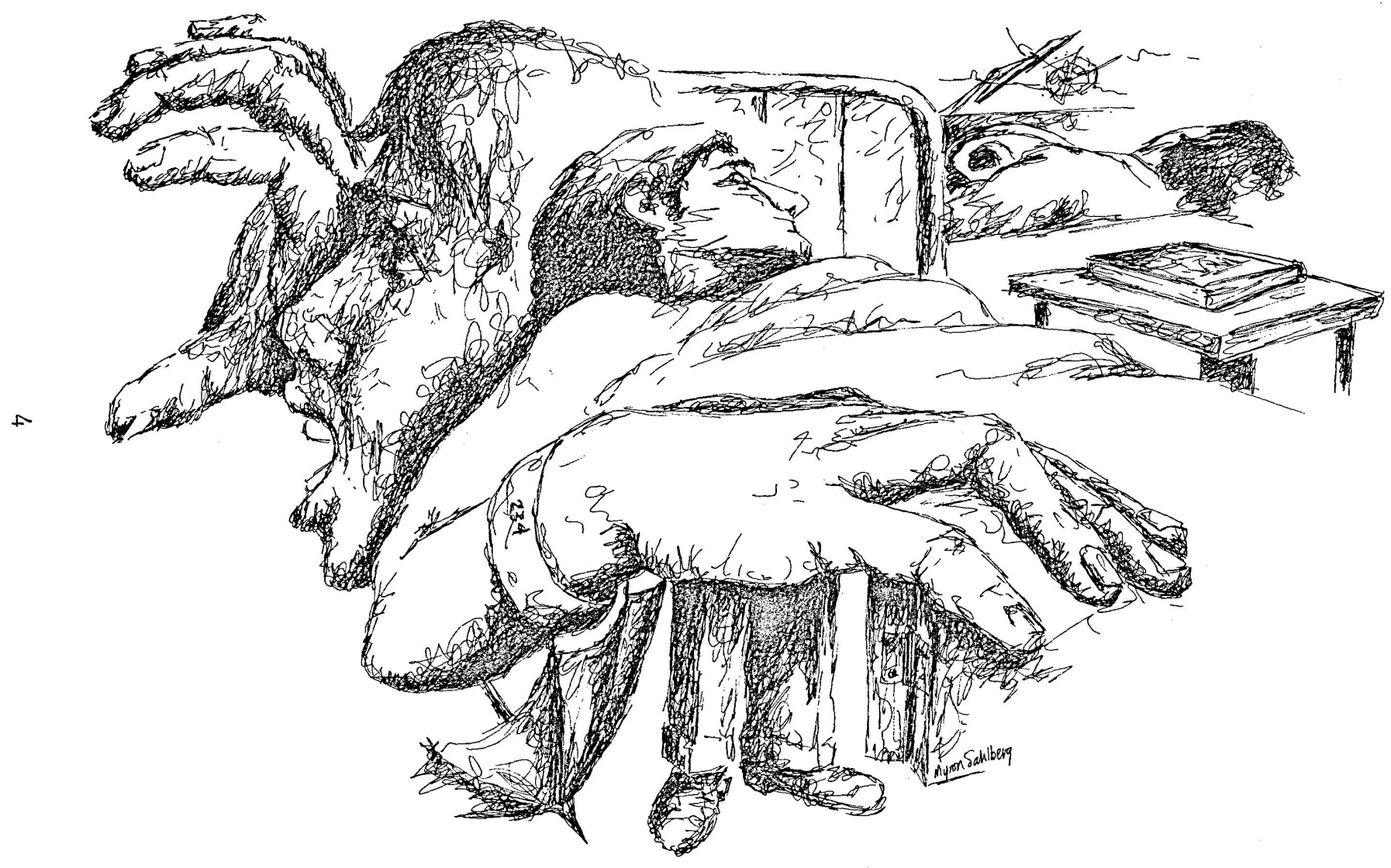




\section{LA VICTIME}

I1 rouvrit les yeux. Une faible lumière entrait par l'unique fenêtre. Dans cette demi-obscurité il ne pouvait distinguer que quelques silhouettes bizarres. Il se demanda où il était, mais avant de trouver une réponse a cette question, il 1'entendit, ce bruit, ce grincement. "Quelqu'un vient a" ...".

En ouvrant la porte il vérifia le numéro: "Oui, 234, c'est ce qu'elle m'a dit." Il entra et le vit, allongé sur le dos, sa ceinture attachée par deux cordes aux contrepoids qui pendaient au-dessus du 1it. Le 1it, à côté de la fenêtre, baignait dans une lueur rouge provenant du dehors. Comme il ne voulait pas le déranger, il s'installa silencieusement dans un fauteuil dans le coin de la chambre et veilla sur lui. Il ne bougeait pas, la tête renversée sur l'oreiller, le cou offert. Il écouta la respiration lente et égale, et au bout d'un moment, il s'approcha du lit pour vérifier qu'il dormait. Tout d'un coup il prit conscience du livre qu'il tenait encore à la main et le déposa sur la petite table de chevet. Puis il sortit.

I1 rouvrit les yeux. I1 l'avait entendu de nouveau, ce bruit, ce grincement, ou peut-être pas. Mais quelque chose l'avait réveillé. En essayant de se redresser il découvrit les cordes et la ceinture qui immobilisaient ses jambes. Il se contenta donc de tourner la tête vers la fenêtre et vit le trottoir désert. Dans 1a rue une voiture, arrêtée devant un feu rouge, attendait. I1 regarda son poignet afin 
de voir 1'heure, mais à la place de sa montre il ne portait qu'un mince bracelet en plastique. Dans $1 \mathrm{a}$ demi-obscurité il ne vit que: "234. Vic. . .".

Ca devait être un dimanche car il avait vu ies gens rentrer de l'église. Comme il faisait beau ce matin-là il y avait beaucoup de circulation. "D'autres comme moi se promènent en voiture," se dit-il. Seul dans l'auto arrêtée devant le feu rouge, il écoutait la radio.

Ce corridor était vaste. A droite il passait toujours devant la même porte entrebaillée. A l'intérieur de la chambre il vit la petite table surmontée d'un bouquet de fleurs, ou d'un panier de fruits. Derrière la table se trouvait un lit défait et vide. A gauche il $n^{\prime} y$ avait qu'un mur peint jaune sans aucun tableau pour interrompre sa monotonie. Puis devant lui, il vit la porte, indentique a celle qu'il venait de voir entrebaillée, mais cette porte était fermée. Il la poussa légèrement.

Ce bruit, ce grincement, de nouveau. "Quelqu' un vient $d^{7}$. . .". Il vit la haute silhouette noire encadrée dans un rectangle de lumière jaune.

"Tiens, enfin tu t'es réveillé. On m'a dit de ne pas rester trop longtemps. J'espère que tu $\mathrm{n}^{\prime}$ as pas trop de ma1 maintenant." En indiquant du doigt le livre sur la table de chevet, il dit: "Je t'ai zipporté ça. Peut-être que demain tu voudras lire."

Le matin en se réveillant il entendit des bruits dans le corridor. De sa porte entr'ouverte il vit des femmes pousser une petite table à roulet= tes, chargée de plateaux. Une femme entièrement vêtue de blanc entra, posa un plateau devant lui, et lui dit: "Bonjour, ça va mieux maintenant, j'espère. $S i$ vous avez besoin de quelque chose, vous n'avez qu'à m'appeler. Je reviendrai avec vos médicaments 
quand vous aurez fini."

I1 mangea tout en examinant 1a chambre. Dans le coin il vit un fauteuil près d'un petit bureau. Un placard occupait 1 'autre mur. A part ces meubles il ne vit que son lit et la fenêtre. Par la fenêtre il vit la rue remplie de circulation. Une file d'autos était arrêtée à un feu rouge.

Non, ce n'était pas une Ford, c'était une Chevrolet bleue foncée. Un homme la conduisait. Pendant qu'il attendait, une fine pluie de printemps commença à tomber. Les gouttes sur le pare-brise l'empêchaient de voir le feu. Il mit en marche les essuie-glace. L'auto avançait imperceptiblement. Son compagnon continuait à parler: ". . . rentrer après-demain. J'espère qu'elle n'aura pas d'autres -. Mais qu'est-ce que c'est?"

Elle rentra apportant une petite tasse en papier sur un plateau. Elle lui versa de l'eau de la petite cruche sur la table de chevet à côté $\mathrm{du}$ lit, et la lui tendit. Il 1'avala. En attendant le sommeil qui allait revenir, il chercha lelivre et 1 'ouvrit. Il se mit à lire que cela devait être un dimanche car il avait vu les gens rentrer de l'église. c'était une belle matinée de printemps et il y avait beaucoup de circulation. Son compagnon, assis à côté de lui, se mit à parler. Il arrêta $1^{\prime}$ auto dans une file de voitures à un feu rouge. Devant son auto il en vit une autre, une Ford noire. Une Chevrolet bleue foncée était arrêtée derrière eux. E1le avançait imperceptiblement comme si le chauffeur ne faisiat pas attention. Sans doute qu'il parlait à l'autre occupant de I'auto, une femme. Son compagnon parlait de sa femme: "On m'a dit qu'elle pourra rentrer après-demain. J'espère qu'elle n'aura pas d'autres ennuis. C'est difficile, tu sais, avec . . Mais qu'est-ce que c'est?

De nouveau il entendit ce bruit, ce grincement, 
plus fort cette fois. C'était comme si quelqu'un heurtait un obstacle métallique qui lui résistait.

Il entra et le vit, allongé sur le dos, le buste un peu redressé, la taille enveloppée d'une ceinture blanche attachée par deux cordes aux contrepoids qui pendaient au-dessus du pied du 1 it. "Bonjour. On m'a dit que je te trouverais ici, mais j'ai eu du mal. Toutes les chambres ici se ressemblent tellement, tu sais. Comment vas-tu aujourd' hui?"

Le médicament commençant à agir il ne pouvait plus très bien distinguer les mots. Il ferma le livre et le mit de côté car il s'intéressait à 1 'homme qui venait d'entrer. Il était grand, noir, et portait à la main un parapluie, qu'il avait sécoué en entrant, de sorte que quelques gouttes de pluie étaient tombées sur le sol. Il s'était installé dans le fauteuil dans le coin de la chambre.

Les essuie-glace effaçaient les gouttes. Il appuya sur le frein pour arrêter 1'auto. Dans la Ford noire il vit les deux passagers qui se parlaient. La Chevrolet bleue avançait lentement. Son compagnon continuait à parler. Puis ils $1^{\prime}$ entendirent, ce bruit, ce grincement.

Elle entra avec une seringue hypodermique à la main. "N'ayez pas peur, je ne vous ferai pas mal. Retournez-vous un peu, s'il vous plait." II sentit la chaleur monter dans ses veines et se retourna. I1 la vit sortir laissant la porte entr'ouverte. Par 1'ouverture il ne vit qu'un mur jaune.

II se leva et s'approcha du lit. I1 ne dormait pas, mais ne semblait pas être conscient de la présence de 1'autre. Un plateau avec les 


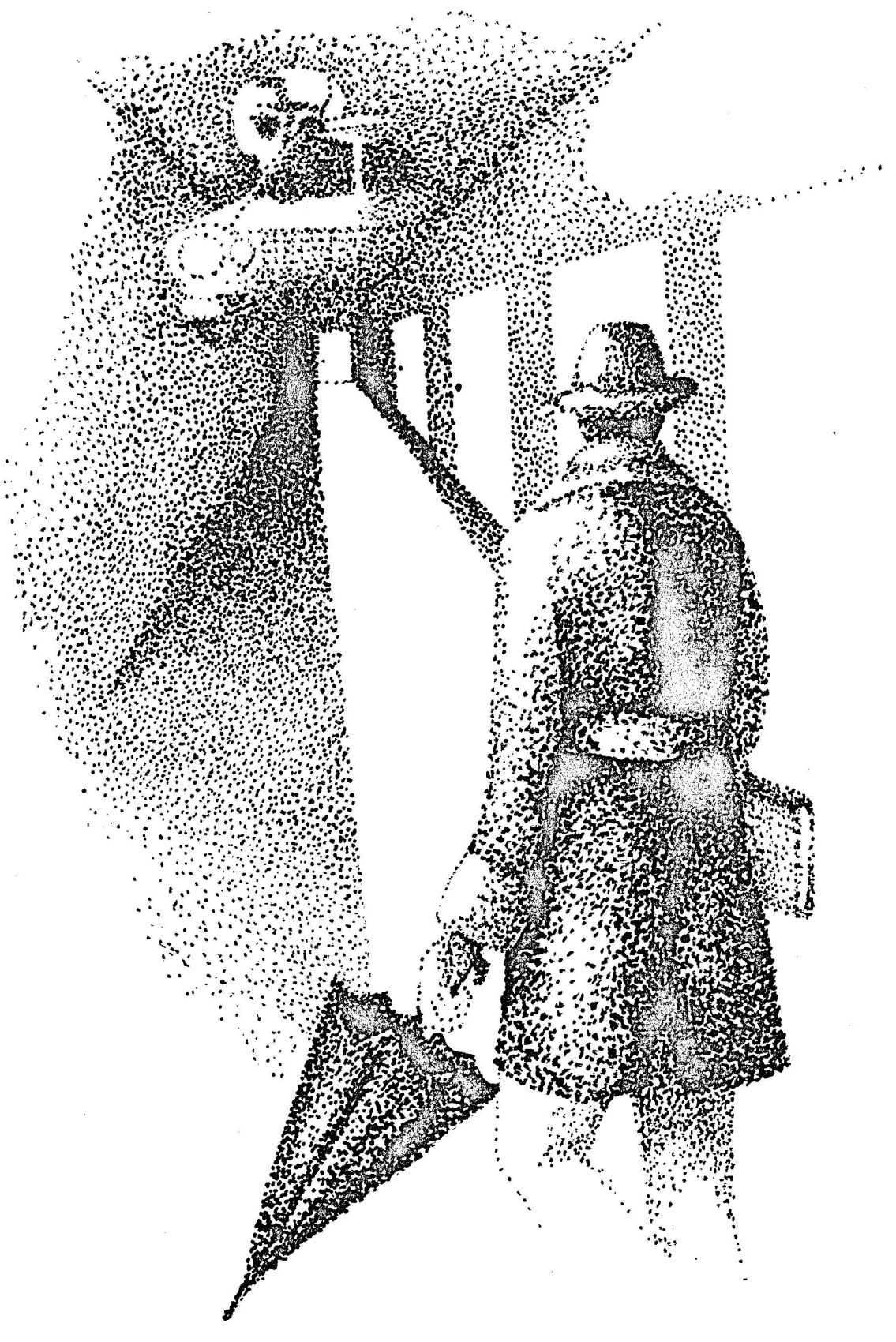


restes du petit déjeuner était sur la table de chevet, à l'endroit où il avait laissé le livre. Par la fenêtre il vit la chaussée mouillée. Quelques voitures attendaient à un feu rouge. Le parapluie qu'il avait appuyé contre le mur à côté du fauteuil tomba. Il le ramassa et sortit.

Ce bruit, ce grincement, comme si on heurtait un obstacle métallique qui résistait, augmenta. La ceinture de sécurité 1 'empêchait de se retourner pour en chercher la cause. Son compagnon se retournait en demandant: "Mais qu'est-ce que c'est?" Le feu restait toujours rouge. Les deux occupants de la Ford noire s'étaient retournês et le regardaient. Dans le rêtroviseur il vit la Chevrolet bleue qui avançait. Il appuya sur le frein pour arrêter 1 'auto.

La femme en blanc ( 1 a même?) rentra et enleva le plateau. Avant de s'en aller elle tira les rideaux. A travers la mince étoffe il vit le trottoir désert. Quelques voitures passaient dans la rue.

Elle avançait de plus en plus. Les roues chassaient sur la chausêe mouillée. Le chauffeur devait essayer de freiner. La Chevrolet bleue et ses occupants étaient projetés en avant. La distance entre les deux voitures diminuait de plus en plus. Son compagnon demanda, en se retournant: "Mais qu'est-ce que c'est?" II répondit: "Quelqu'un vient $d^{\prime}$. . .".

II rouvrit les yeux. 